\title{
Sea piracy sequelae: assessment according to the Diagnostic and Statistical Manual of Mental Disorders-5
}

\author{
Antonio Rosario Ziello ${ }^{1}$, Angiola Maria Fasanaro ${ }^{2}$, Cristina Petrelli ${ }^{1}$, Giovanna Ricci ${ }^{3}$, \\ Ascanio Sirignano ${ }^{3}$, Francesco Amenta ${ }^{1,4}$ \\ ${ }^{1}$ Centre for Clinical Research, Telemedicine and Telepharmacy, University of Camerino, Camerino, Italy \\ ${ }^{2}$ Neurology Unit, National Hospital “A. Cardarelli”, Napoli, Italy \\ ${ }^{3}$ School of Law, Legal Medicine Section, University of Camerino, Camerino, Italy \\ ${ }^{4}$ Research Department, International Radio Medical Centre (CIRM), Roma, Italy
}

\begin{abstract}
Background: Our previous studies have investigated the psychological consequences of kidnapping in a group of Italian seafarers assaulted by sea pirates and held in captivity and in their family members by the criteria of the Diagnostic and Statistical Manual of Mental Disorders (DSM)-4. These studies have shown that both the victims and the family members showed significant psychological disturbances, corresponding to a chronic Post-Traumatic Stress Disorder (PTSD), in the victims, and a pattern of anxiety and depression in their family members. After publication of these studies, an updated edition of the DSM became available, namely, the DSM-5. The DSM-5 redefines some diagnostic criteria, including those related to the PTSD. This work was focused on the re-evaluation of the results of our previous studies in the light of the DSM-5 diagnostic criteria.

Materials and methods: Sixteen Italians including 4 kidnapped seafarers and 12 family members were examined by a semi-structured interview followed by Clinician-Administered PTSD Scale (CAPS-DX) and the Cognitive Behaviour al Assessment (CBA 2.0) for victims and by State-Trait Anxiety Inventory (STAI) X-1 and X-2 of CBA 2.0 and the Hamilton Depression Rating Scale (HDRS) for family members. Data already obtained were reviewed and re-analysed according to the DSM-5 criteria and the Clinician-Administered PTSD Scale for DSM-5 (CAPS-5).

Results: The use of the CAPS-5 did not modify the diagnosis for the victims' group: 3 of 4 had a PTSD diagnosis performed through the CAPS-5. Seven of 12 family members had PTSD diagnosis performed through the CAPS-5, with negative cognitions and mood symptoms being those obtaining the highest score. Conclusions: Using DSM-5 criteria, the diagnosis of PTSD in the direct victims of piracy was confirmed. The same diagnosis could apply to a group of their family members. Besides anxiety and fear, in fact, we found in 7 out 12 subjects the presence of symptoms included by the DSM-5 in the PTSD spectrum. These symptoms were: avoidance, negative alterations in mood and cognition, blame of self or others. The use of updated diagnostic criteria may enable more correct assessment of the consequences of piracy acts. This may be also useful for establishing proper compensations for the damage suffered by seafarers, depending on the degree of disability resulting from the criminal acts they suffered.
\end{abstract}

(Int Marit Health 2014; 65, 4: 230-234)

Key words: maritime piracy, victims, relatives, DSM-5, psychopathology 


\section{INTRODUCTION}

The Diagnostic and Statistical Manual of Mental Disorders (DSM) contains the updated guidelines for the diagnosis of mental health disorders and is widely recognised as a tool to which professionals in the field should refer. Its last edition, the DSM-5 [1], published in May 2013, shows some differences from the DSM-4 [2, 3]. These differences are related to some diagnostic criteria, and include the Post-Traumatic Stress Disorder (PTSD). This is a chronic and debilitating condition, that may develop after unexpected and very dangerous life events [4].

Changes of PTSD diagnostic criteria involve primarily 3 aspects. First, the clinical presentation of PTSD, which includes not only anxiety and fear, but also dysphoria, anhedonia, guilt, shame, anger, and aggressiveness [4]. As a consequence, PTSD is no more classified within the Anxiety Disorders, but in the more specific category of Stress Disorders. A second difference lies in the more accurate definition of the traumatic event, which must be "sudden and catastrophic", and leading to the PTSD not only if directly experienced by the subject, but also if it occurs to "a close family member or friend". Third, the DSM-5 indicates that symptoms as avoidance, negative alterations in mood and cognition, blaming oneself or others, reckless or self-destructive behaviour are frequently found in PTSD.

In 2 previous studies we have investigated the psychological consequences of kidnapping in a group of Italian seafarers assaulted by sea pirates and held in captivity for long time and in their close family members [5, 6]. These studies have shown that both the victims and the family members showed significant psychological disturbances, corresponding in the victims to a chronic PTSD, and in their family members to a pattern of anxiety and depression.

In this paper we re-evaluated our results in the light of the DSM-5. The purpose of the present work was to assess if (a) the diagnosis of PTSD applied to the victims was coherent with the DSM-5, and if (b) the diagnosis of PTSD might be applied to the family members as well. Piracy causes sufferance to seafarers, who have been affected by this type of criminality, and their families [7] for which they may be entitled to compensation. Objective assessment of the damage they suffered can provide the basis for the development of compensation plans appropriate to the damage suffered.

\section{MATERIALS AND METHODS}

Participants in our analysis were 16 Italians included in 2 previous studies on psychological consequences in victims of maritime piracy and their families $[5,6]$. They were divided into 2 groups. The "victims group" consisted of 4 kidnapped seafarers. The mean of days of captivity was $265 \pm 72.12$ days. The "families group" included
12 relatives ( 8 females and 4 males) living in the household of kidnapped seafarers. All subjects were evaluated at home or in another place they indicated and were examined face to face by a psychologist at the $20^{\text {th }}$ week subsequent to victims release.

A semi-structured interview was used as a research instrument, followed by specific questionnaires, after the consent for the treatment of data had been subscribed. This initiative of the International Radio Medical Centre (CIRM), the Italian Telemedical Maritime Assistance Service was held under the auspices of the Ministry of Infrastructure and Transport. CIRM established a team, called Psychological Emergency Piracy (PEP) to ease the discomfort of the victims of piracy acts and their families. PEP team approached victims who were submitted to the Clinician-Administered PTSD Scale (CAPS-DX) and the Cognitive Behavioural Assessment (CBA 2.0). The CAPS-DX, structured clinical interview [8], considers 17 symptoms of PTSD grouped into 3 clusters according to DSM-4: intrusive recollection, avoidance/numbing and hyperarousal symptoms. Moreover, CAPS-DX includes aspects related to the traumatic event, such as feelings of guilt, loss of emotional involvement in environmental events, derealisation and depersonalisation. The CBA 2.0 provides a general overview of the psychological problems in the individual and social domain [9]. This scale evaluates: the temporary emotional state of an individual in a particular situation, defined as "state anxiety" (State-Trait Anxiety Inventory - STAI X-1); the almost stable characteristic of personality, defined as "trait anxiety" (State-Trait Anxiety Inventory - STAI X-2); the different dimensions of the personality (Eysenck Personality Questionnaire reduced form - EPQ/R) that is articulated into 4 sub-scales (introversion - EPQ/R-E; emotional instability - EPQ/R-N; social maladjustment - EPQ/R-P; lie - EPQ/R-L); the somatic disorders (Questionnaire Psychophysiological - QPF/R); the specific fears (Inventory of Fears - IP/R); the depressive symptoms (Questionnaire D - QD); and the presence of intrusive thoughts and compulsive behaviours (Maudsley Obsessional-Compulsive Questionnaire - MOCQ/R).

Each family member was interviewed first by a semi-structured approach and then examined using the 2 subscales STAI X-1 and X-2 of CBA 2.0 and the Hamilton Depression Rating Scale (HDRS) [10]. The HDRS (also known as Ham-D) is the most widely used clinician-administered depression assessment scale. The 21 trait items of this scale investigates the presence and severity of depression by probing mood, feelings of guilt, suicide ideation, insomnia, agitation, anxiety, weight loss and somatic symptoms [10].

The results, which are reported in detail elsewhere $[5,6]$ may be summarised as follows: 3 victims had developed PTSD and 1 had developed Adjustment Disorder, while 6 of 


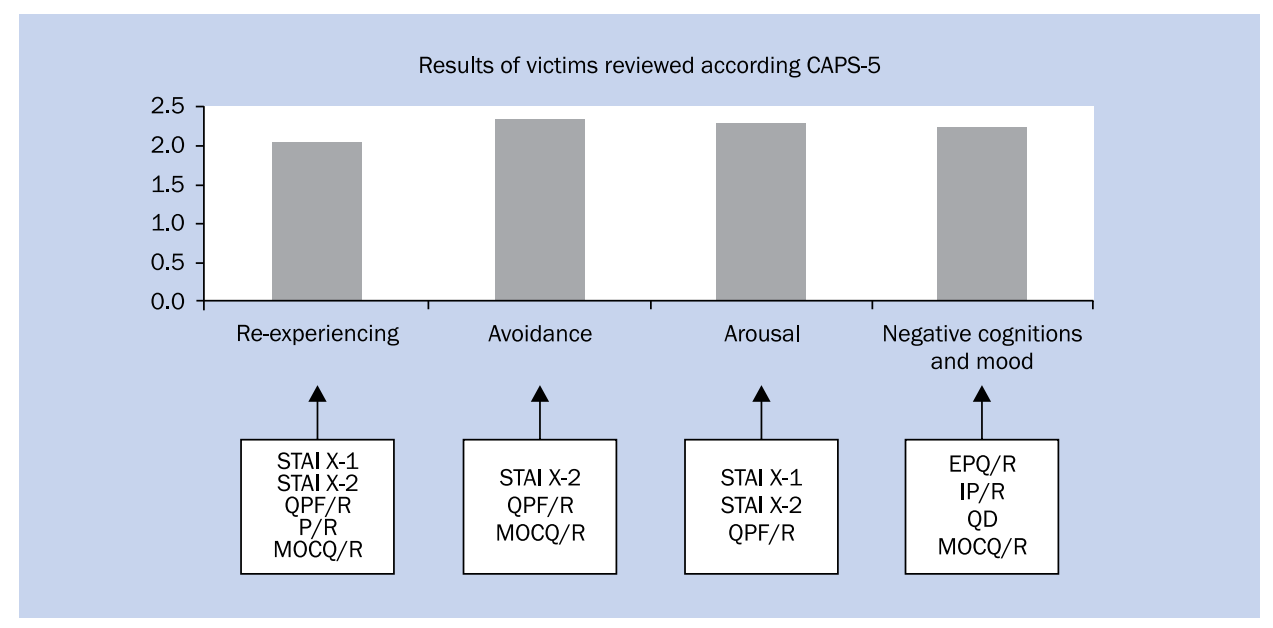

Figure 1. Severity of Post-Traumatic Stress Disorder (PTSD) symptoms in victims of piracy acts assessed by the Clinician-Administered PTSD Scale for DSM-5 (CAPS-5)

the family members had also developed anxiety and 4 depression (the association of both problems was found in 2).

The data was reviewed and recorded following the DSM-5 criteria and the Clinician-Administered PTSD Scale for DSM-5 (CAPS-5), which represents the gold standard in PTSD assessment currently [4]. The changes from previous CAPS for DSM-4 are: it requires the identification of a single index trauma to serve as the basis of symptom inquiry; it is composed by 30-item questionnaire, corresponding to the DSM-5 diagnosis for PTSD; the items are rated with a single severity score in contrast to previous versions of the CAPS which required separate frequency and intensity scores.

\section{RESULTS}

First, the structures of CAPS-DX, CBA 2.0 and HDRS, used in the previous studies were analysed, in order to identify the sub-scales providing information to assess the new criteria of DSM-5. For this phase a working group including clinical psychologists, medical doctors (neurologists) and legal medicine specialists were involved as a part of the clearing process. The following sub-scales were used to assess the victims with the CAPS-5: STAI X-1, STAI X-2, QPF/R, IP/R and MOCQ/R for "re-experiencing"; STAI X-2, QPF/R and MOCQ/R for "avoidance"; STAI X-1, STAI X-2 and QPFR/R for "arousal”; EPQ/R, IP/R, QD and MOCQ/R for "negative cognitions and mood". The degree of agreement was $91 \%$

The use of the CAPS- 5 did not modify the diagnosis for the victims group: 3 of 4 had a PTSD diagnosis performed through the CAPS-5. Severity of the symptoms were also considered. Those of avoidance were most severe (M $2.38 \pm$ \pm 0.46 ), in agreement with previous findings [4]. Figure 1 shows severity means of the symptoms.
The following sub-scales were used to assess the families with the CAPS-5: STAI X-1, STAI X-2 and HDRS for "re-experiencing"; STAI X-2 and anamnestic data for "avoidance"; STAI X-1, STAI X-2 and HDRS for "arousal"; HDRS and anamnestic data for "negative cognitions and mood". The degree of agreement was $83 \%$. Seven of 12 family member had PTSD diagnosis performed through the CAPS-5. Negative cognitions and mood obtained the highest score (2.45 \pm \pm 0.41 ) in terms of severity of the symptoms (Fig. 2).

\section{DISCUSSION}

A large and recent literature has been reviewed by the authors of the DSM- 5 in order to formulate updated criteria for the diagnosis of different psychological disorders, including the PTSD $[4,11,12]$. The new edition of the diagnostic criteria of PTSD has included some emotional items. Instead of 3 major symptom clusters for PTSD, the DSM- 5 now lists 4 clusters. Differently from its previous version DSM-4, the DSM-5 indicates that, following a sudden and catastrophic event, multiple negative emotions may be triggered, leading to the PTSD, including, but not limited to, anxiety and fear. These emotions include other aspects such as the feeling of guilt, shame and anger and play a relevant role in the development and maintenance of PTSD [4]. These aspects may be found not only in the victims but also in their family members. This observation suggests that also family members of victims of piracy merit assessment of the mental health and symptoms they can develop. We have therefore evaluated these aspects in the family members of the victims of maritime piracy. Our analysis has shown that subjects showing distress were affected by the multiple and diverse emotional symptoms that allowed the diagnosis of PTSD. These symptoms are included in a wide spectrum, which reflects the different psychological vulnerabilities of 


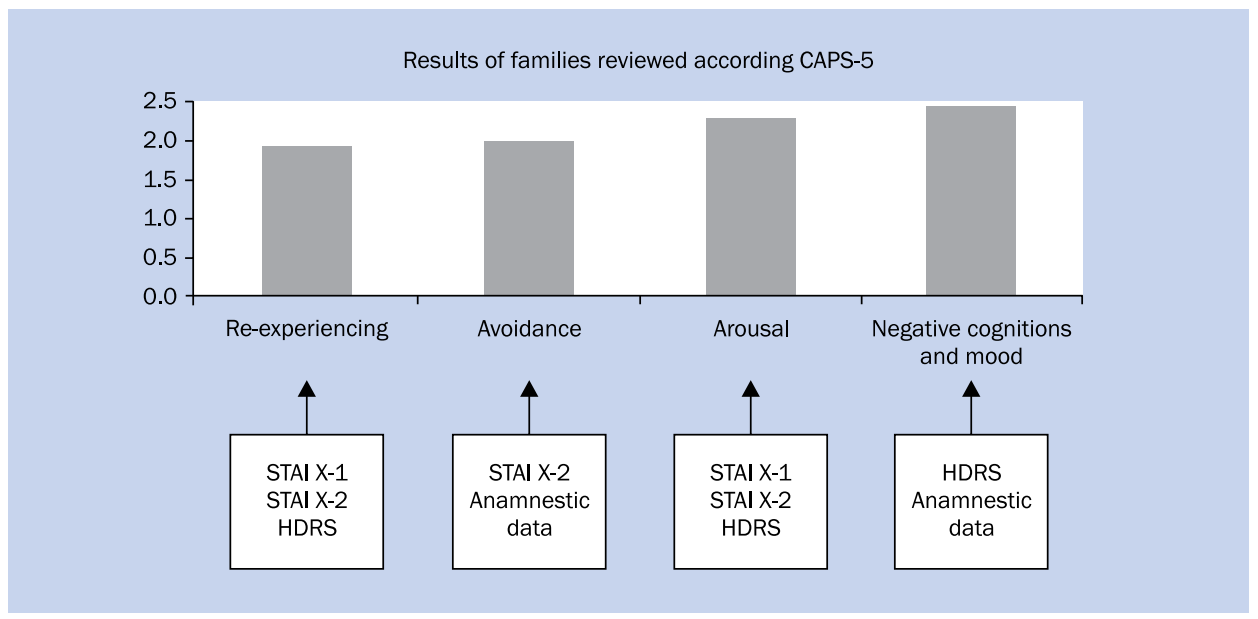

Figure 2. Severity of symptoms in family members of victims of piracy acts

each individual. Hence, the traumatic event may ultimately create different phenotypes of the PTSD [4]. This broad spectrum of symptoms allows the diagnosis of PTSD in the family members where a more simplistic diagnosis of anxiety and depression had been previously done $[13,14]$. Another aspect underlined in the DSM-5 is the inclusion in the PTSD of those subjects who, in spite of not been involved directly, are close family member of the victims, such as in our cases.

The presence of some previous psychological disturbances does not exclude the diagnosis of PTSD, as, following the DSM-5, the trauma may also act in worsening an already present frailty. This is the case of one of our subjects, where symptoms became more severe after the traumatic event, which represented an additional factor of distress in a person already affected by psychological disturbances.

\section{CONCLUSIONS}

In conclusion, when applying the DSM-5 criteria, we were able to confirm the diagnosis of PTSD in the direct victims of piracy. The same diagnosis could apply to a group of their family members. Besides anxiety and fear, in fact, we found in 7 out 12 subjects the presence of symptoms included by the DSM-5 in the PTSD spectrum. These symptoms were avoidance, negative alterations in mood and cognition, blaming oneself or others.

Does this re-evaluation have any practical impact? We do think so. PTSD treatment in fact is known to be different from that of anxiety disorder, and its early recognition would be more beneficial in terms of future wellbeing. Its correct diagnosis, thus, would impact favourably on the outcome. This work is also an example of how new consensus within the psychiatric community can change the picture of a disease. Global piracy at sea is now decreasing, due to the international crackdown on pirate gangs off the coast of Somalia, however there are reports of an increased risk in other areas, particularly on West African coast. Pirates, moreover, far from being "the enemies of all" as Marcus Tullius Cicero called them, seem nowadays connected to political instability and war lords.

If seafarers victims of piracy acts and their families are entitled to compensation and outstanding wages, this should be covered by their contracts. In piracy cases there are usually doubled wages for sailing in the piracy area, while compensation for loss of personal effects and all injury and death benefits are also doubled. Different countries and social security agencies are regulating these problems parallel with the occurrence and increase of piracy phenomenon. The Security Council of the United Nations has invited all States, along with the International Maritime Organisation and other relevant international organisations, to adopt or recommend measures to prevent maritime hijackings, as well as to protect seafarer victims of piracy and to assist them after their release [15]. In spite of this, information on the consequences that piracy may cause on the mind and behaviour of victims of these acts is still sparse and a scientific approach to the evaluation of their effects is mainly empiric.

Analysis of psychological and psychiatric consequences of piracy using the most updated version of the reference manual used primarily by clinicians in the United States to diagnose mental disorders such as the DSM-5 [1] may represent a reference for the development of rehabilitation strategies and compensation plans of victims. Our study has shown that a significant percentage of family members of seafarers developed PTSD. This observation deserves further study and suggests the need to extend neuropsychological protection to victim families as well. Compensation for work-related pathologies interests almost exclusively the employee. However, the peculiarity of mental disorders 
caused by piracy in their family should also consider this issue for the fair compensation to the workers involved.

\section{ACKNOWLEDGEMENTS}

The present study was supported by a grant of the Italian Ministry of Infrastructure and Transport (General Directorate for Maritime and Inland Waterways). Patience and collaboration of victims of piracy acts examined in this study and their families are gratefully acknowledged.

\section{REFERENCES}

1. American Psychiatric Association. Diagnostic and Statistical Manual of Mental Disorders (DSM- $5^{\circledR}$ ) Psychiatric Publishing, Arlington, Virginia 2013.

2. Kapfhammer HP. Trauma and stressor-related disorders: diagnostic conceptualization in DSM-5. Nervenarzt 2014; 85: 553-563.

3. Rodríguez-Testal JF, Senín-Calderón C, Perona-Garcelán S. From DSM-4-TR to DSM-5: Analysis of some changes. Int J Clin Health Psychology 2014; 14: 221-231.

4. Weathers FW, Marx BP, Friedman MG, Schnurr PP. Posttraumatic Stress Disorder in DSM-5: New criteria, new measures, and implications for assessment. Psychol Inj Law 2014; 7: 93-107.

5. Ziello AR, Degli Angioli R, Fasanaro AM, Amenta F. Psychological consequences in victims of maritime piracy: the Italian experience. Int Marit Health 2013; 64: 136-141.
6. Ziello AR, Degli Angioli R, Fasanaro AM, Amenta F. Psychological distress in families of victims of maritime piracy: the Italian experience. Int Marit Health 2014; 65: 28-32.

7. Hurlburt K, Seyle DC. The human cost of maritime piracy 2012. Ocean Beyond Piracy 2013 Broomfield, Colorado.

8. Blake DD, Cook JD, Keane TM. Post-traumatic stress disorder and coping in veterans who are seeking medical treatment. J Clin Psychol 1992; 48: 695-704.

9. Le scale CBA. Cognitive Behavioural Assessment: un modello di indagine psicologica multidimensionale; Raffaello Cortina; Milano 2002.

10. Hamilton M. A rating scale for depression. J Neurol Neurosurg Psychiatry 1960; 23: 56-62.

11. Levin AP, Kleinman SB, Adler JS. DSM-5 and posttraumatic stress disorder. J Am Acad Psychiatry Law 2014; 42: 146-158.

12. Kirkpatrick HA, Heller GM. Post-traumatic stress disorder: theory and treatment update. Int J Psychiatry Med 2014; 47: 337 $-346$.

13. Friedman MJ. Finalizing PTSD in DSM-5: getting here from there and where to go next. J Traumatic Stress 2013; 26: 548-556.

14. Kilpatrick DG, Resnick HS, Milanak ME, Miller MW, Keyes KM, Friedman MJ. National estimates of exposure to traumatic events and PTSD prevalence using DSM-4 and DSM-5 criteria. J Traumatic Stress 2013; 26: 537-547.

15. United Nations Security Council. Delegations in security council note progress in combating piracy, but warn 'pirates will quickly be back in their skiffs' if attention diverted. 19 November 2012. http://www. un.org/press/en/2012/sc10820.doc.htm. 